\title{
The Selective Prostacyclin (Ip) Receptor Agonist Selexipag: A Promising Drug in Pediatric Pulmonary Artery Hypertension
}

\author{
Martin Koesten berger ${ }^{1 *}$, Hannes Sall mon ${ }^{2}$ and Georg Hansmann ${ }^{3}$ \\ ${ }^{1}$ Division of Pediatric Cardiology, Department of Pediatrics, Medical University Graz, Austria \\ ${ }^{2}$ Department of Neonatology, Charité - Universitätsmedizin Berlin, Germany \\ ${ }^{3}$ Department of Pediatric Cardiology and Critical Care, Hannover Medical School, Hannover, Germany
}

${ }^{*}$ Corresponding author: Martin Koestenberger, Department of Pediatrics, Medical University Graz, Auenbruggerplatz Graz, Austria, Tel: 43316385 84276; Fax: 43316385 13682; E-mail: martin.koestenberger@medunigraz.at; georg.hansmann@gmail.com

Received date: October 18, 2017; Accepted date: November 03, 2017; Published date: November 10, 2017

Citation: Koestenberger M, Sallmon H, Hansmann G (2017) The Selective Prostacyclin (Ip) Receptor Agonist Selexipag: A Promising Drug in Pediatric Pulmonary Artery Hypertension. J Intensive \& Crit Care. Vol.3 No.4:42.

\section{Introduction}

Pulmonary artery hypertension (PAH) is still an important cause of morbidity and mortality in children. Despite recent developments in $\mathrm{PH}$-specific therapies, survival of patients with idiopathic PAH (IPAH) remains poor and appears to be worse in children compared with adults. During the past few years, treatment of PAH has undergone a real evolution, which has led to the current approval by regulatory agencies of 10 drugs for adult patients from three main pharmacological groups and four different routes of administration (oral, inhaled, subcutaneous and intravenous). Additional drugs such as the selective prostacyclin (IP) receptor agonist selexipag are expected to be approved in the near future. However, emerging therapeutic strategies for adult $\mathrm{PH}$, such as a triple oral combination therapy, have not been sufficiently studied in children. The use of selexipag in children with IPAH, or pulmonary artery hypertension (PAH) associated with congenital heart disease (PAH-CHD) is of interest. Selexipag is the first orally administered prostacyclin receptor agonist with a non-prostanoid structure [1]. Selexipag leads to a vasodilation and a decrease in proliferation. An active metabolite of selexipag, ACT-333679, shows a high selectivity to the IP receptor, and its long half-life enables a twice-a-day oral dosing regimen [2,3]. As of December 2015 selexipag (Uptravi) was FDA approved in the US, making it the first oral prostacyclin agonist to be used in adults with PAH.

Herein, we discuss the sparse available data on the use of selexipag in pediatric populations, which is currently limited on only a few children with IPAH and PAH-CHD. At the time of publication a PubMed search using the term "Selexipag" retrieved a total of 74 publications, by adding the term "pediatric" only 3 publications were present. While currently used off-label, some centers administer this promising drug to pediatric patients as part of an oral combination therapy including the more common therapies phosphodiesterase 5 inhibitors (PDE-5i) and endothelin receptor antagonists (ERA).

Several countries participated in a double blind, placebocontrolled Phase III study on selexipag, with 1,156 adult PAH patients (GRIPHON study) [4]. The primary endpoint was a composite of death from any cause or a complication related to
PAH up to the end of the treatment period. The risk of the primary composite endpoint of death or a complication related to PAH was significantly lower with selexipag than with placebo. Although more than $70 \%$ of the patients were receiving one or more PAH therapeutic drugs, and about $30 \%$ were treated with double combination therapy (an ERA and a PDE-5 inhibitor), the risk of morbidity/mortality events decreased by $40 \%$ in the selexipag group as compared with that in the other groups [4]. The effect of selexipag in the GRIPHON trial was consistently confirmed in all pre-specified subgroups such as combined use to other PAH therapeutic drugs, NYHA/WHO functional class, age, and PAH disease etiology. In detailed analyses, the effect of selexipag was evident already at early stage and was maintained throughout the trial period [4]. Connective tissue diseaseassociated pulmonary arterial hypertension (PAH-CTD) has a poorer prognosis as compared to other $\mathrm{PH}$ etiologies. The underlying CTD is thought to influence treatment response and outcomes. Recently, a subgroup analysis from the GRIPHON trial [4] showed that Selexipag delayed progression of PAH and was well-tolerated among PAH-CTD patients, including those with PAH due to a systemic lupus erythematodes [5]. The efficacy of selexipag was seen by improvements in pulmonary hemodynamics, exercise capacity, and clinical symptoms in a cohort of adult Japanese PAH patients [6]. More detailed discussion and information about selexipag use in adult PAH can be found in several recent reviews [7-14].

Pediatric data on selexipag in $\mathrm{PAH}$ to date are very sparse. Here, we report the initial experience in 6 pediatric patients on selexipag from 2 centers, which includes one case that was the first published pediatric case worldwide, describing clinical and hemodynamic improvements by cardiac catheterization in a 12 year old female with hereditary PAH [15]. In addition, Gallotti et al. [16] recently published their experience of using the selective prostacyclin (IP) receptor agonist selexipag in 5 children with either IPAH or PAH-CHD. Four children had IPAH and were 3, 7, 8, 17 years old, and one was diagnosed with $\mathrm{PAH}-\mathrm{CHD}$ and was 10 years old. Both groups emphasized the need for a detailed evaluation of children with $\mathrm{PAH}$, by applying the gold standard cardiac catheterization and a non-invasive diagnostic method, i.e., parameters determined with transthoracic echocardiography $[15,16]$. Pediatric inpatients with PAH, ill- 
appearing PAH patients, and those with hemodynamics suggesting "high risk" are usually started on a PDE-5i and ERA simultaneously (upfront combination), in accordance with the most recent consensus statements [17]. Prior to the start of an additional third agent (i.e., intravenous treprostinil, epoprostenol or oral selexipag), all of their patients were on PDE5i and ERA dual therapy. The authors discuss transitioning patients on a continuous treprostinil infusion from intravenous therapy to an oral prostanoid therapy, presenting a wellconducted protocol with selexipag being up titrated while treprostinil is weaned [16]. The rationale for transitioning from intravenous treprostinil to oral selexipag is the concern for central venous line infection in the REVEAL registry (approx. 1 after 3,000 treatment days), however, innovative subcutaneously implanted, tunneled, central intravenous catheter pumps appear to have much lower complication rates including line infection [18].

In pediatric $\mathrm{PH}$ there is a clear need for comprehensive cardiac catheterization in children with $\mathrm{PH}$, especially in those who fail to respond to pharmacotherapy or those who receive an additional PAH-targeted drug, be it off-label or not $[19,20]$. We have to mention that possible indications for the use of selexipag in pediatric PAH - beyond transitioning from intravenous prostacyclin analogue to oral selexipag - might be expanded by primarily adding selexipag as a third oral PAHtargeted agent in patients not responding to dual therapy. The concept of add-on oral selexipag to dual oral combination therapy (mostly PDE5i and ERA) is powered by the idea of (1) avoiding central venous lines, especially in small children but also in teenagers or in patients who deny this approach, and/or (2) to achieve stabilization, and if necessary as a bridge to bilateral lung transplantation or reverse Potts shunt creation $[19,20]$.

Selexipag was also used in one child with single ventricle physiology before and/or after their total cavopulmonary anastomosis [16], however, it should be realized that this is per se a high-risk population, and selexipag may worsen oxygenation by opening intrapulmonary arterio-venous shunts. Therefore, at the moment we currently see no favorable indication/risk profile for using this agent in such pediatric patients.

Although promising, the rare data on oral selexipag in children with PAH [15-17], however, do not allow any conclusions regarding short-, mid- or long-term efficacy. Authors did find that oral selexipag was safe in 5 children with $\mathrm{PAH}$ and that transitioning from parenteral to oral prostanoid in children can be pursued safely under a strict protocol, while another group described the initiation of oral triple therapy with selexipag in a child with severe PAH [16]. Given the fact that first clinical data in PAH children are now available $[15,16]$, oral selexipag use will increase over the next months and years in the pediatric age group [21]. This is supported by a recent bio-comparison study investigating a pediatric tablet (containing only $50 \mu$ g selexipag) in adults [22]. In healthy male adults either the normal adult starting dose of $200 \mu \mathrm{g}$ or 4 tablets of a (future) $50 \mu \mathrm{g}$ pediatric preparation were administered. The authors found that pharmacokinetic characteristics of selexipag and its metabolite ACT-333679 were comparable in both groups [22].
In that context, we emphasize that the add-on use of oral selexipag must still be considered "experimental therapy", and suggest at best strict patient selection and enrollment in a clinical study (currently in preparation), including regular echocardiographic evaluations [23] plus cardiac catheterization before and 6 months after the start of selexipag, as previously described [15]. Given that efficacy of selexipag was confirmed in adult PAH patients in the GRIPHON trial [4], this medication, although mentioned as a possible add-on therapy in current pediatric guidelines [24], is recommended only in adults as combination therapy with an ERA and/or a PDE-5 inhibitor in adults but not in children with PAH.

We want to address the need for a careful and systematic therapeutic approach in children with PAH. The now available sparse pediatric experience on the use of an oral selective prostacyclin receptor agonist $[15,16]$, with more such studies ahead, is promising. Thus, the future decision to add selexipag as a third oral PAH agent or to replace intravenously administered PAH drugs with oral selexipag in stable patients, may become feasible in children with significant PAH.

\section{References}

1. Hardin EA, Chin KM (2016) Selexipag in the treatment of pulmonary arterial hypertension: Design, development and therapy. Drug Des Devel Ther 10: 3747-3754.

2. Asaki T, Kuwano K, Morrison K, Gatfield J, Hamamoto T, et al. (2015) Selexipag: An oral and selective IP prostacyclin receptor agonist for the treatment of pulmonary arterial hypertension. J Med Chem 58: 7128-7137.

3. Duggan ST, Keam SJ, Burness CB (2017) Selexipag: A review in pulmonary arterial hypertension. Am J Cardiovasc Drugs 17: 73-80.

4. Sitbon O, Channick R, Chin KM, Frey A, Gaine S, et al. (2015) Selexipag for the treatment of pulmonary arterial hypertension. $\mathrm{N}$ Engl J Med 373: 2522-2533.

5. Gaine S, Chin K, Coghlan G, Channick R, Di Scala L, et al. (2017) Selexipag for the treatment of connective tissue diseaseassociated pulmonary arterial hypertension. Eur Respir J 50: 1602493.

6. Tanabe N, Ikeda S, Tahara N, Fukuda K, Hatano M, et al. (2017) Efficacy and safety of an orally administered selective prostacyclin receptor agonist, selexipag, in Japanese patients with pulmonary arterial hypertension. Circ J 81: 1360-1367.

7. Baker WL, Darsaklis K, Singhvi A, Salerno EL (2017) Selexipag, an oral prostacyclin-receptor agonist for pulmonary arterial hypertension. Ann Pharmacother 51: 488-495.

8. Bruderer S, Hurst N, Remenova T, Dingemanse J (2017) Clinical pharmacology, efficacy, and safety of selexipag for the treatment of pulmonary arterial hypertension. Expert Opin Drug Saf 16: 743-751.

9. Noel ZR, Kido K, Macaulay TE (2017) Selexipag for the treatment of pulmonary arterial hypertension. Am J Health Syst Pharm 74: $1135-1141$

10. Ghosh RK, Ball S, Das A, Bandyopadhyay D, Mondal S, et al. (2017) Selexipag in pulmonary arterial hypertension: Most updated evidence from recent pre-clinical and clinical studies. J Clin Pharmacol 57: 547-557. 
11. Jain S, Khera R, Girotra S, Badesch D, Wang Z, et al. (2017) Comparative effectiveness of pharmacologic interventions for pulmonary arterial hypertension: A systematic review and network meta-analysis. Chest 151: 90-105.

12. Richter MJ, Gall H, Grimminger J, Grimminger F, Ghofrani HA (2016) Selexipag for the treatment of pulmonary arterial hypertension. Expert Opin Pharmacother 17: 1825-1834.

13. O'Connell C, Amar D, Boucly A, Savale L, Jaïs X, et al. (2016) comparative safety and tolerability of prostacyclins in pulmonary hypertension. Drug Saf 39: 287-294.

14. Sørensen $L M$, Wehland $M$, Krüger $M$, Simonsen $U$, Nassef $M Z$, et al. (2017) A special focus on selexipag treatment of pulmonary arterial hypertension. Curr Pharm Des.

15. Geerdink LM, Bertram H, Hansmann G (2017) First-in-child use of the oral selective prostacyclin IP receptor agonist selexipag in pulmonary arterial hypertension. Pulm Circ 7: 551-554.

16. Gallotti R, Drogalis-Kim DE, Satou G, Alejos J (2017) Single-center experience using selexipag in a pediatric population. Pediatr Cardiol.

17. Hansmann G, Apitz C (2016) treatment of children with pulmonary hypertension. expert consensus statement on the diagnosis and treatment of paediatric pulmonary hypertension. The European Paediatric Pulmonary Vascular Disease Network, endorsed by ISHLT and DGPK. Heart 102: 67-85.

18. Ewert R, Richter MJ, Steringer-Mascherbauer R, Grünig E, Lange TJ, et al. (2017) Intravenous treprostinil infusion via a fully implantable pump for pulmonary arterial hypertension. Clin Res Cardiol.

19. Hansmann G, Apitz C, (2016) The need for comprehensive cardiac catheterization in children with pulmonary hypertension. J Am Coll Cardiol 67: 1009-1010.

20. Hansmann G (2017) Pulmonary hypertension in infants, children and young adults. J Am Coll Cardiol 69: 2551-2569.

21. Koestenberger M, Hansmann G (2017) future applications of the selective prostacyclin (ip) receptor agonist selexipag in pediatric pulmonary hypertension. Pediatr Cardiol.

22. Boehler M, Bruderer S, Ulč I, Dingemanse J (2017) Biocomparison study of adult and paediatric dose strengths of the prostacyclin receptor agonist selexipag. Eur J Drug Metab Pharmacokinet.

23. Koestenberger M, Apitz C, Abdul-Khaliq H, Hansmann G (2016) Transthoracic echocardiography for the evaluation of children and adolescents with suspected or confirmed pulmonary hypertension. Expert consensus statement on the diagnosis and treatment of paediatric pulmonary hypertension. The european paediatric pulmonary vascular disease network, endorsed by ISHLT and DGPK. Heart 102: 14-22.

24. Hansmann G, Apitz C, Abdul-Khaliq H, Alastalo TP, Beerbaum P, et al. (2016) Executive summary. Expert consensus statement on the diagnosis and treatment of paediatric pulmonary hypertension. The European Paediatric Pulmonary Vascular Disease Network, endorsed by ISHLT and DGPK. Heart 102: 86-100. 